\title{
Study of dry eye in post-menopausal women
}

\author{
Samuel Cornelius Gnanadurai J. ${ }^{1}$, S.Vimala Karunanidhi ${ }^{2}$, Balaji Ramraj ${ }^{3}$, Sathish Kumar M. ${ }^{4}$, Swamyraj S.V. $^{5}$
}

${ }^{1}$ Dr. J. Samuel Cornelius Gnanadurai, Associate Professor, Department of Ophthalmology, ${ }^{2}$ Dr. S. Vimala Karunanidhi, Senior Resident, Department of Ophthalmology, ${ }^{3}$ Dr. Balaji Ramraj, Associate Professor, Department of Community Medicine, ${ }^{4}$ Dr. M. Sathish Kumar, Resident PG, Department of Ophthalmology, ${ }^{5}$ Dr. S.V. Swamyraj, Professor \& HOD, Department of Ophthalmology, 1,2,3,4,5 Authors affiliated with SRM Medical College Hospital and Research Centre, Chennai, Tamil Nadu, India.

Corresponding Author: Dr. J. Samuel Cornelius Gnanadurai, M.B.B.S., M.S. (Ophthal), DNB, F.I.C.O., Associate Professor, Department of Ophthalmology, SRM Medical College Hospital and Research Centre, Chennai, Tamil Nadu, India. E-mail: samueldurai@yahoo.com

\begin{abstract}
Aim: The aim of the study is to evaluate hospital-based prevalence of dry eye in post-menopausal women, according to age, symptoms of dry eye and occupation. Materials and Methods: A cross-sectional study of 200 post-menopausal women attending ophthalmology out-patient department of SRM Medical College Hospital for 6 months. The diagnosis of dry eye was based on Schirmer's test. The value of $<5 \mathrm{~mm}$ is considered severe dry eye, $5-8$ mm is moderate dry Eye, $9-14 \mathrm{~mm}$ is mild dry eye and $>15 \mathrm{~mm}$ is normal. The results were analyzed using the Chi-square test. Results: The prevalence of dry eye in post-menopausal women was $52 \%$. The prevalence of dry eye increases with age from $26.8 \%$ (50-54 years) to $85.7 \%$ (>70years). The prevalence of dry eye in patients with symptoms of dry eye was $88 \%$ and without symptoms was $30.4 \%$. The Schirmer's test showed majority of patients had mild dry eye (27.5\%) followed by moderate dry eye in $21 \%$. The prevalence of dry eye with outdoor occupation was $64.4 \%$ compared to $41.8 \%$ for indoors. Conclusion: The prevalence of dry eye in this study was high. Even patients without symptoms of dry eye can have subclinical dry eye. Patients with outdoor occupation had a higher prevalence of dry eye. Simple treatment with artificial tear substitutes can effectively manage mild and moderate dry eye and help post-menopausal women in improving their quality of life. It would be advisable to screen all post-menopausal women for dry eye using a simple Schirmer`s test.
\end{abstract}

Keywords: Dry eye, Post-menopausal women, Schirmer`s test, Tear film.

\section{Introduction}

Dry Eye is defined as "A disorder of the tear film due to tear deficiency or excessive tear evaporation which causes damage to the inter-palpebral ocular surface and is associated with symptoms of ocular discomfort [1]." Dry eye is a multifactorial disease of the ocular surface characterized by a loss of homeostasis of the tear film, and accompanied by ocular symptoms, in which tear film instability and hyperosmolarity, ocular surface inflammation and damage, and neurosensory abnormalities play etiological roles [2].

Hyperosmolarity can result in symptoms of ocular discomfort, dryness and vision disturbances. There are two main types of Dry eye, namely Aqueous deficient dry eye and Evaporative dry eye. Aqueous deficient dry

Manuscript received: $28^{\text {th }}$ August 2019

Reviewed: $8^{\text {th }}$ September 2019

Author Corrected: $15^{\text {th }}$ September 2019

Accepted for Publication: $19^{\text {th }}$ September 2019 eye is defined by the presence of normal evaporation with a reduced tear volume and is associated with lacrimal gland dysfunction. Evaporative dry eye is characterized by a normal tear volume with an increased rate of tear evaporation and is usually associated with meibomian gland dysfunction. Dry eye is not a disease entity, but a symptom complex which occurs due to deficiency or abnormalities of tear film.

The symptoms of dry eye are eye dryness, discomfort, sensitivity to light, itching, burning, tearing, grittiness, discomfort, fatigue and visual disturbances [3]. Dry eyes if left untreated can increase the risk of bacterial and viral infection of eye. It has been consistently found that dry eye increases with age and disproportionately affects women especially after menopause. Studies have shown that estrogen receptor mRNA are present in the lacrimal gland, meibomian gland, lids, palpebral and 


\section{Original Research Article}

bulbar conjunctiva, cornea and anterior ocular surface. Therefore, reduction in naturally occurring estrogen after menopause may increase the chances of developing dry eye in post-menopausal women.

Estrogen deficiency may lead to sebaceous gland alteration, so further destabilization of the tear film due to meibomian gland dysfunction.

Low androgen levels in old age are most consistently associated with dry eye. With age there is increase in meibomian gland dropout, particularly above the age of 50 years, which increases the chances of meibomian gland dysfunction.

It is important to understand the inter-relationship between Dry eye disease and women's health which would be useful not only to ophthalmologists but also to general physicians and gynecologists in the management of post-menopausal disorders.

The current study was done with the aim to study the prevalence of dry eye in post-menopausal women attending the ophthalmology department.

\section{Materials and Methods}

Aim of study: The aim of the study is to evaluate hospital based prevalence of dry eye in postmenopausal women, prevalence of dry eye in postmenopausal women according to age, prevalence of dry eye in post-menopausal women with and without symptoms of dry eye and prevalence of dry eye according to occupation.

Study Design: This is a cross-sectional study of postmenopausal women attending ophthalmology outpatient department of SRM Medical College Hospital for a period of 6 months.

Sample size: 200 post-menopausal women.
Diagnostic procedure: The diagnosis of dry eye was based on Schirmer's test. All the subjects will undergo Schirmer's test which is a screening test for dry eye. Special Standardized filter paper strips were placed inside the lower eye lid of both eyes. The subjects were asked to close their eyes for five minutes and the paper was removed after five minutes.

The amount of moisture on the filter strips was noted immediately. In order to measure only the basal secretion of tears, anesthetic proparacaine hydrochloride eye drops were administered five minutes prior to testing. This ensured that there was no reflex tearing due to the insertion of Schirmer's strip into the eye.

Scoring system: The value of $<5 \mathrm{~mm}$ is considered severe dry eye, $5-8 \mathrm{~mm}$ is moderate dry eye, $9-14$ $\mathrm{mm}$ is mild dry eye and $>15 \mathrm{~mm}$ is normal. Patients with reading $<15 \mathrm{~mm}$ were taken as those having Dry eye disease. Schirmer's test will be done for all the subjects.

Statistical analysis: The prevalence of Dry eye will be calculated. The Schirmer's test values will be compared between the groups. The results will be analyzed using the Chi-square test.

Inclusion criteria: All women who attained natural menopause were included in the study.

Exclusion criteria: Women with pre-existing Dry eye or Ocular surface disorder, history of ocular trauma or ocular surgery, patients on long term ocular topical medications, any systemic disease associated with generalized dryness and Contact lens users were excluded from the study.

Ethics: Clearance was obtained from scientific and ethical committee of SRM Medical College Hospital and Research Centre for the study. Written and informed consent was obtained from all patients in English and local language.

\section{Results}

Table-1: Prevalence of Dry eye

\begin{tabular}{|c|c|}
\hline Patients & 200 (total) \\
\hline Dry eye & $104(52 \%)$ \\
\hline
\end{tabular}

A total of 200 post-menopausal women took part in this study out of which 104 women had dry eye. The prevalence of dry eye in this study was $52 \%$. 
Table-2: Distribution of dry eye according to age.

\begin{tabular}{|c|c|c|}
\hline Age Group & No. of patients & Patients with dry eye \\
\hline $50-54$ & 41 & $11(26.8 \%)$ \\
\hline $55-59$ & 72 & $35(48.6 \%)$ \\
\hline $60-64$ & 55 & $32(58.1 \%)$ \\
\hline $65-69$ & 18 & $14(77.7 \%)$ \\
\hline$>70$ & 14 & $12(85.7 \%)$ \\
\hline
\end{tabular}

Chi square value $-22.75 ; \mathrm{P}$ value -0.0001 statistically significant

The majority of patients in this study were seen in the age group of 55-59 years (72 patients), 60-64 years (55 patients) and 50-54 years (41 patients). It was noted that the prevalence of dry eye increases with age from $26.8 \%$ (50-54 years), $48.6 \%$ (55-59 years), $58.1 \%$ (60-64 years), $77.7 \%$ (65-69 years) to $85.7 \%$ ( $>70$ years). The prevalence of Dry eye increased with advancing age.

Table-3: Relationship between dry eye and symptoms.

\begin{tabular}{|c|c|c|}
\hline & With Symptoms & Without Symptoms \\
\hline No. of patients & 75 & 125 \\
\hline Patients with dry eye & $66(88 \%)$ & $38(30.4 \%)$ \\
\hline
\end{tabular}

Chi square value $-62.31 ; \mathrm{P}$ value -0.0001 statistically significant

The prevalence of dry eye in patients with symptoms of dry eye was $88 \%$ and in patients without symptoms of dry eye was $30.4 \%$. It was noted that 38 patients who did not have any symptoms of dry eye had subclinical dry eye which was detected with Schirmer`s test.

Table-4: Schirmer's test grading of dry eye.

\begin{tabular}{|c|c|c|c|}
\hline Schirmer's test & Grade & No. of patients & Percentage \\
\hline$>15 \mathrm{~mm}$ & Normal & 96 & $48 \%$ \\
\hline $9-14 \mathrm{~mm}$ & Mild & 55 & $27.5 \%$ \\
\hline $5-8 \mathrm{~mm}$ & Moderate & 42 & $21 \%$ \\
\hline$<5 \mathrm{~mm}$ & Severe & 7 & $3.5 \%$ \\
\hline
\end{tabular}

Out of the 104 patients with dry eye, 55patients had mild dry eye, 42 patients had moderate dry eye and 7 patients had severe dry eye. Majority of patients in this study had mild dry eye (27.5\%) followed by moderate dry eye in $21 \%$.

Table-5: Relationship between dry eye and occupation.

\begin{tabular}{|c|c|c|c|}
\hline Occupation & Normal & Dry eye & Total \\
\hline Outdoor & $32(35.5 \%)$ & $58(64.4 \%)$ & 90 \\
\hline Indoor & $64(58.1 \%)$ & $46(41.8 \%)$ & 110 \\
\hline
\end{tabular}

Chi square value $-10.15 ; \mathrm{P}$ value -0.001 statistically significant

In this study, it was observed that the prevalence of dry eye among post-menopausal women with outdoor occupation was $64.4 \%$ compared to $41.8 \%$ for women who stayed indoors. Dry eye was more among working women compared to housewives. 


\section{Discussion}

Tears help to maintain a normal healthy ocular surface as well as providing a smooth refractive surface for optimal vision. A crucial factor in dry eye is the loss of tear film stability which leads to tear film hyperosmolarity. Hyperosmolarity results in symptoms of ocular discomfort, dryness, foreign body sensation, grittiness, photophobia and blurred vision.

Hyperosmolarity of the tear film can result from either reduced tear volume (Aqueous deficient dry eye) or increased tear evaporation (evaporative dry eye). Dry eye can lead to corneal complications like punctate epithelial erosions, mucus plaques, corneal infiltrates, corneal thinning and peripheral vascularization [4]. Slit lamp examination and Schirmer's test are very simple, easy to do and sensitive in the diagnosis of dry eye. In population-based studies, it has been consistently found that dry eye increases with age.

There is a age related decrease in meibomian gland secretion possibly due to atrophy of acinar cells as well as alteration in the quality of meibomian gland secretions. The lacrimal gland also appears to undergo age related changes leading to decrease in aqueous production. Dry eye has been found to disproportionately affect women [5]. The higher prevalence of dry eye in women is attributed to levels of sex steroids (androgens and estrogens) and hormonal changes following menopause, differences in metabolism, lifestyle, response to diagnostic and therapeutic interventions as well as differences in immunity [6].

Low androgen levels are consistently associated with dry eye. The levels of 17- $\beta$-estradiol, estrone and testosterone in severe dry eye show inverse correlation with tear film osmolarity. There is a significant decrease in levels of cortisol, 4-androstene-3, 17-dione, and 17 $\alpha$ hydroxyprogesterone in patients with dry eye.

In the present study, the prevalence of dry eye in postmenopausal women was $52 \%$. The majority of patients in this study were seen in the age group of 5559 years (72 patients) and 60-64 years (55 patients). It was noted that the prevalence of dry eye increases with age from $26.8 \%$ in the $50-54$ age group to $85.7 \%$ in the $>70$ age group. The prevalence of dry eye in patients with symptoms of dry eye was $88 \%$ and in patients without symptoms of dry eye was $30.4 \%$. Therefore, even patients without symptoms of dry eye can have subclinical dry eye which can easily be diagnosed with the Schirmer's test. The Schirmer's test showed majority of patients in this study had mild dry eye $(27.5 \%)$ followed by moderate dry eye in $21 \%$. In this study, it was observed that the prevalence of dry eye among post-menopausal women with outdoor occupation was $64.4 \%$ compared to $41.8 \%$ for women who stayed indoors. Patients with outdoor occupation had a higher prevalence of dry eye probably due to air pollution, low humidity, high temperature and sunlight exposure.

A similar study by Rahul Agarwal et al [7] who studied 300 post- menopausal women found hospital-based prevalence of drye eye in postmenopausal women to be $32 \%$ and another study by Gokul Vinod Kumar et al [8] found the prevalence of dry eye in a tertiary care centre to be $39.5 \%$. M.R. Pujari et al [9] found dry eye to be more common in the 65-75 years age group and more common in rural population. Mohana Majumdar et al [10] studied 293 post-menopausal women and found the common ocular findings were foreign body sensation, grittiness, hyperemia, mucoid discharge and fluctuating or blurry vision.

The prevalence of dry eye in post-menopausal women with symptoms of dry eye in this study was $82.97 \%$. Rahul Verma et al [11] found that $46 \%$ had mixed type of dry eye, $22 \%$ had tear film instability and $6 \%$ had aqueous deficient dry eye. Cynthia Matossian et al [12] found that women with autoimmune diseases like Sjogren's syndrome, Systemic lupus erythematosus, Rheumatoid arthritis, Rosacea, Hashimoto`s thyroiditis and Graves' disease had higher chances of developing dry eye. Also, women who used contact lenses or had undergone any ocular procedure like LASIK, blepharoplasty, botulinum toxin injection and cosmetic eyelid surgery had higher chances of developing dry eye. A study done by Caterina Gagliano et al [13] suggests that deficiency in sexual hormones may cause not only a reduction in tear production leading to aqueous deficient dry eye, but also a dysfunction in meibomian gland function causing an evaporative dry eye.

Early detection of dry eye is important to arrest the progression of dry eye as well as to prevent the complications that can reduce corneal transparency and reduce vision. Environmental and behavioral modifications such as taking breaks while reading, keeping the computer monitors at eye level, use of UV protective glasses and humidification of the environment can be tried. Tear film substitutes such as 
hydroxy propyl methyl cellulose, carboxyl methyl cellulose, polyvinyl alcohol, chondroitin sulphate and sodium hyaluronate are the mainstay of dry eye management [14]. Anti-inflammatory therapy such as cyclosporine eye drops and low-dose corticosteroids such as loteprednol etabonate eye drops are considered to interrupt the inflammatory cascade.

Systemic Omega-3 fatty acids are also used. Severe dry eye needs surgical management like punctal occlusion with silicone plugs. Hormone replacement therapy [15] can also be considered in post-menopausal women with dry eye. Newer techniques like biomarkers of dry eye disease, image analysis software to evaluate tear smoothness and stability non-invasively would provide better diagnosis of dry eye.

\section{Conclusion}

The prevalence of dry eye in postmenopausal women in this study was high $(52 \%)$. The prevalence of dry eye increases with age from $26.8 \%$ in the $50-54$ age group to $85.7 \%$ in the $>70$ age group. The prevalence of dry eye in patients with symptoms of dry eye was $88 \%$ and in patients without symptoms of dry eye was $30.4 \%$. Therefore, even patients without symptoms of dry eye can have subclinical dry eye.

The Schirmer's test showed majority of patients in this study had mild dry eye $(27.5 \%)$ followed by moderate dry eye in $21 \%$. Patients with outdoor occupation had a higher prevalence of dry eye $(64.4 \%)$. Simple treatment with artificial tear substitutes can effectively manage mild and moderate dry eye and help postmenopausal women in improving their quality of life. It would be advisable to screen all postmenopausal women for dry eye using a simple Schirmer`s test.

\section{What the study adds to the existing knowledge?}

The study has shown that prevalence of dry eye in postmenopausal women is high and increases with age and outdoor exposure.

A simple Schirmer's test screening for all postmenopausal women will help to detect even subclinical dry eye. Simple treatment with artificial tear substitutes can treat dry eye and improve the quality of life of postmenopausal women.

\section{Author's contributions}

Dr. J. Samuel Cornelius Gnanadurai: Concept, study design and manuscript preparation.
Dr.S. Vimala Karunanidhi: Scientific and ethical committee presentation and patient selection.

Dr. Balaji Ramraj: Statistical analysis.

Dr. M. Sathish Kumar: Data Collection

Dr. S.V. Swamyraj: Data collection and proof approval.

Funding: Nil, Conflict of interest: Nil

Permission from IRB: Yes

\section{References}

1. The definition and classification of dry eye disease: report of the Definition and Classification Subcommittee of the International Dry Eye Work Shop (2007). Ocul Surf. 2007;5(2):75-92. doi: https://doi. org/ 10. $1016 / \mathrm{S} 1542-0124(12) 70081-2$

2. Peck T, Olsakovsky L, Aggarwal S. Dry Eye Syndrome in Menopause and Perimenopausal Age Group. J Midlife Health. 2017; 8(2):51-54. doi: 10. 4103/jmh.JMH_41_17.

3. Uchino M, Schaumberg DA. Dry Eye Disease: Impact on Quality of Life and Vision. Curr Ophthalmol Rep. 2013;1(2):51-57. doi:10.1007/s40135-013-0009-1

4. Aditi G, Surabhi S. Study of Dry Eyes in PostMenopausal Women-A Rural Hospital Based Study. Int J Adv Res Ideas Innov Technol. 2017;3(1):473-478.

5. Adlakha N, Tirkey ER, Lakhtakia S. To assess the prevalence of dry eye disease in postmenopausal females in a tertiary care centre in Central India. J Med Sci Clin Res. 2017;05(10):29012-29017.

6. Moss SE, Klein R, Klein BE. Prevalence of and risk factors for dry eye syndrome. Arch Ophthalmol. 2000;118(9): 1264-1248. doi:10. 1001/archopht. 118. 9.1264

7. Agarwal R, Singh P, Rajpal T, Kumar R, Raghuwanshi S, Ramnani V. Hospital based study of prevalence of dry eye in post-menopausal women. Indian J Clinic Experiment Ophthalmol. 2016;2(1):5661. doi:10.5958/2395-1451.2016.00014.7

8. Kumar GV, Praneetha G, Pandharpurkar A, Prasad B, Pavani G, Vasavi T, Naik A. A study on prevalence of dry eyes among menopausal women attending a tertiary care centre in Hyderabad, Telangana. International J Commun Med Publ Health. 2018;6(1):423-427. doi: http://dx.doi.org/10.18203/2394-6040.ijcmph201 85266 


\section{Original Research Article}

9. Pujari MR, Salagar K, Bagare SN. Prevalence of Dry Eye in post-menopausal women. J Evolution Med Dent Sci.2015;4(75):13005-13010. doi:10.14260/jemds /2015 / 1874.

10. Mohana Majumdar, Rekha Khandelwal, Tanya Gangwani. Comparison of dry eyes in post-menopausal women with and without symptoms of dry eyes. J Evolution Med Dent Sci. 2014;3(57):12933-12938. doi:10.14260/jemds/2014/3716.

11. Rahul Verma, Mishrikotkar JP, Thakre S. Comparitive study of occurrence of dry eye in post menopausal women. Medpulse Int med J.2016;3(10): 893-896.

12. Cynthia Matossian, Marguerite McDonald, Kendall E. Donaldson et al. J Women's Health.2019;28(4):502514. doi:10.1089/jwh.2018.7041.
13. Gagliano C, Caruso S, Napolitano G, Malaguarnera G, Cicinelli MV, Amato R, et al. Low levels of 17 - $\beta$ oestradiol, oestrone and testosterone correlate with severe evaporative dysfunctional tear syndrome in postmenopausal women: a case-control study. $\mathrm{Br} \mathrm{J}$ Ophthalmol 2014; 98(3): 371-376 .doi: 10.1136/ bjophthalmol- 2012-302705.

14. Huang FC, Tseng SH, Shih MH, Chen FK. Effect of artificial tears on corneal surface regularity, contrast sensitivity, and glare disability in dry eyes. Ophthalmol. 2002; 109 (10):1934-1940. doi: 10. 1016 /s0161- 6420(02)01136-3

15. Schaumberg DA, Buring JE, Sullivan DA, Dana MR. Hormone replacement therapy and dry eye syndrome.JAMA. 2001;286(17):2114-2119.doi:10.1001 / jama. 286. 17.2114

\section{How to cite this article?}

Samuel Cornelius Gnanadurai J, S.Vimala Karunanidhi, Balaji Ramraj, Sathish Kumar M, Swamyraj S.V. Study of dry eye in post-menopausal women. Trop J Ophthalmol Otolaryngol.2019;4(5):328-333.doi: 10.17511/jooo.2019.i05.04 